\title{
Properties for Nonlinear Fractional SubLaplace Equations on the Heisenberg Group
}

WANG Xinjing and NIU Pengcheng*

Department of Applied Mathematics, Northwestern Polytechnical University, Xi'an 710129, China.

Received 5 June 2018; Accepted 23 November 2018

\begin{abstract}
The aim of the paper is to study properties of solutions to the nonlinear fractional subLaplace equations on the Heisenberg group. Based on the method of moving planes to the Heisenberg group, we prove the Liouville property of solutions on a half space and the symmetry and monotonicity of the solutions on the whole group respectively.
\end{abstract}

AMS Subject Classifications: 35A01, 35J57, 35D99

Chinese Library Classifications: 0175.2

Key Words: Heisenberg group; fractional subLaplace equation; method of moving planes.

\section{Introduction}

In this paper, we consider the fractional subLaplacian

$$
\left(-\Delta_{\mathbb{H}}\right)^{s} u(\xi)=C_{Q, s} P V \int_{\mathbb{H}^{n}} \frac{u(\xi)-u(\eta)}{\left|\eta^{-1} \circ \xi\right|_{\mathbb{H}} Q+2 s} \mathrm{~d} \eta
$$

on the Heisenberg group $\mathbb{H}^{n}$, where $0<s<1, Q=2 n+2, C_{Q, s}$ is a positive constant and $P V$ is the Cauchy principle value, and study properties of cylindrical solutions to the equation

$$
\left(-\Delta_{\mathbb{H}}\right)^{s} u(\xi)=u^{p}(\xi) \quad \text { in } \mathbb{H}_{+}^{n}=\left\{\xi \in \mathbb{H}^{n} \mid t>0\right\},
$$

where $1<p<\infty$, and another equation

$$
\left(-\Delta_{\mathbb{H}}\right)^{s} u(\xi)=f(u) \quad \text { in } \mathbb{H}^{n},
$$

*Corresponding author. Email addresses: pengchengniu@nwpu.edu.cn (P. C. Niu), shingw@sina.com (X. J. Wang) 
where $f$ is Lipschitz continuous.

Recall that the fractional Laplacian in $\mathbb{R}^{n}$ is a nonlocal pseudodifferential operator defined by

$$
(-\Delta)^{\alpha} u(x)=C_{n, \alpha} \lim _{\varepsilon \rightarrow 0} \int_{\mathbb{R}^{n} \backslash B_{\varepsilon}(x)} \frac{u(x)-u(y)}{|x-y|^{n+2 \alpha}} \mathrm{d} y,
$$

where $0<\alpha<1, C_{n, \alpha}$ is a constant, $u$ belongs to the Schwartz space, the limit stands for the Cauchy principle value. Since the nonlocal property of the operator $(-\Delta)^{\alpha}$ brings new difficulties to investigate, Caffarelli and Silvestre in [1] put forward the extension method which can reduce the nonlocal problem relating to $(-\Delta)^{\alpha}$ to a local one in higher dimensions. This method has been applied to deal with equations involving the fractional Laplacian and fruitful results were obtained, see [2] and the references therein. But the method has tha limitation that $\frac{1}{2} \leq \alpha<1$. Chen, Li and Li [4] developed a direct method of moving planes to handle the problem containing $(-\Delta)^{\alpha}$ for $0<\alpha<1$ and this direct method has been used successfully to deduce symmetry, monotonicity and nonexistence for many fractional Laplace equations, see [3,4] and references therein. Recently, Wang and Niu [5] considered (1.2) in $\mathbb{H}^{n}$, and showed properties of solutions.

To the elliptic equation

$$
-\Delta u=f(u) \quad \text { in } \mathbb{R}^{n},
$$

Gidas, Ni and Nirenberg [6] proved that the positive solutions are radial symmetric with the assumptions that the limitation of $u$ is zero at the infinity. In $\mathbb{H}^{n}$ we can also give a similar result of [6] to the equation (1.3).

There are many interesting results about subLaplace equations on the Heisenberg group (see [7-11]. It appears several different definitions of the fractional power subLaplacian in $\mathbb{H}^{n}$ (see [12-14] etc.). The definition of fractional power subLaplacian in Roncal and Thangavelu [14] is indeed a generalization of Cowling and Haagerup [15] about the Heat semigroup. The fractional power subLaplace equations can also be studied by generalizing the extension method in [1] to $\mathbb{H}^{n}$, for example, see $[13,16,17]$. Extending the method of moving planes in $[3,4,18]$ to $\mathbb{H}^{n}$, we establish the Liouville type result of the solution to (1.2) on $\mathbb{H}_{+}^{n}=\left\{\xi \in \mathbb{H}^{n} \mid t>0\right\}$, and the symmetric and monotone of the solution to (1.3) on $\mathbb{H}^{n}$.

Our main results are the following:

Theorem 1.1. Let $0<s<1$ and $1<p<\infty$. If $u \in L_{2 s}\left(\mathbb{H}_{+}^{n}\right) \cap C_{\text {loc }}^{1,1}\left(\mathbb{H}_{+}^{n}\right)$ is a nonnegative cylindrical solution to

$$
\begin{cases}\left(-\Delta_{\mathbb{H}}\right)^{s} u(\xi)=u^{p} & \xi \in \mathbb{H}_{+}^{n}, \\ u(\xi)=0 & \xi \notin \mathbb{H}_{+}^{n},\end{cases}
$$

with

$$
\lim _{|\xi|_{H} \rightarrow \infty} u(\xi)=0,
$$

and lower semi-continuous on $\overline{\mathbb{H}}_{+}^{n}$, then $u \equiv 0$. 
Theorem 1.2. Let $0<s<1$, and $u \in L_{2 s}\left(\mathbb{H}_{+}^{n}\right) \cap C_{\text {loc }}^{1,1}\left(\mathbb{H}_{+}^{n}\right)$ be a nonnegative cylindrical solution to the equation (1.3) and suppose $u$ satisfies (1.7). If $f$ is Lipschitz continuous, then $u$ must be symmetric and monotone with respect to t about some point in $\mathbb{H}^{n}$.

In this paper, we use an assumption (1.7). Under this condition, be different from [4], we do not need to take advantage of the CR inversion (which is called the Kelvin transform in [4]), and the range of index $p$ can be extended to $(1, \infty)\left(p \in\left(1, \frac{n+2 s}{n-2 s}\right]\right.$ in [4]). Notice that (1.7) also appeared in [6], and Theorem 1.2 is the generalization of result in [6].

The authors in [14] required that $u \in C_{0}^{\infty}\left(\mathbb{H}^{n}\right)$ in (1.1). We point out that (1.1) is also well defined for $u \in L_{2 s}\left(\mathbb{H}^{n}\right) \cap C_{l o c}^{1,1}\left(\mathbb{H}^{n}\right)$, where

$$
L_{2 s}\left(\mathbb{H}^{n}\right)=\left\{u: \mathbb{H}^{n} \rightarrow \mathbb{R} \mid \int_{\mathbb{H}^{n}} \frac{|u(\xi)|}{1+|\xi|_{\mathbb{H}}{ }^{Q+2 s}} \mathrm{~d} \xi<\infty\right\} .
$$

The proof can refer to [5].

The paper is organized as follows. Section 2 collects some well known contents on $\mathbb{H}^{n}$ and three maximum principles which proved in [5]. Based on these principles, the proofs of Theorems 1.1 and 1.2 are derived by using the method of moving planes in Sections 3 and 4 .

\section{Preliminaries}

The Heisenberg group $\mathbb{H}^{n}$ is the Euclidean space $\mathbb{R}^{2 n+1}(n \geq 1)$ endowed with the group law o:

$$
\bar{\xi} \circ \bar{\xi}=\left(x+\bar{x}, y+\bar{y}, t+\bar{t}+2 \sum_{i=1}^{n}\left(x_{i} \bar{y}_{i}-y_{i} \bar{x}_{i}\right)\right),
$$

where $\xi=\left(x_{1}, \cdots, x_{n}, y_{1}, \cdots, y_{n}, t\right):=(x, y, t) \in \mathbb{R}^{n} \times \mathbb{R}^{n} \times \mathbb{R}$ and $\bar{\xi}=(\bar{x}, \bar{y}, \bar{t})$. Denote by $\delta_{\mathcal{K}}$ the dilations on $\mathbb{H}^{n}$, i.e.

$$
\delta_{\kappa}(\xi)=\left(\kappa x, \kappa y, \kappa^{2} t\right), \kappa>0,
$$

which satisfies $\delta_{\kappa}(\bar{\xi} \circ \bar{\xi})=\delta_{\kappa}(\bar{\xi}) \circ \delta_{\kappa}(\xi)$.

The left invariant vector fields corresponding to $\mathbb{H}^{n}$ are

$$
X_{i}=\frac{\partial}{\partial x_{i}}+2 y_{i} \frac{\partial}{\partial t^{\prime}}, \quad Y_{i}=\frac{\partial}{\partial y_{i}}-2 x_{i} \frac{\partial}{\partial t}, \quad i=1, \cdots, n, \quad T=\frac{\partial}{\partial t^{\prime}}
$$

and $X_{i}$ and $Y_{j}$ satisfy

$$
\left[X_{i}, Y_{j}\right]=-4 T \delta_{i j}, \quad\left[X_{i}, X_{j}\right]=\left[Y_{i}, Y_{j}\right]=0, \quad i, j=1, \cdots, n .
$$

The Heisenberg gradient of a function $u$ is defined as

$$
\nabla_{\mathbb{H}} u=\left(X_{1} u, \cdots, X_{n} u, Y_{1} u, \cdots, Y_{n} u\right)
$$


and the subLaplacian $\Delta_{\mathbb{H}}$ on $\mathbb{H}^{n}$ is

$$
\Delta_{\mathbb{H}}:=\sum_{i=1}^{n}\left(X_{i}{ }^{2}+Y_{i}^{2}\right)=\sum_{i=1}^{n}\left(\frac{\partial^{2}}{\partial x_{i}{ }^{2}}+\frac{\partial^{2}}{\partial y_{i}{ }^{2}}+4 y_{i} \frac{\partial^{2}}{\partial x_{i} \partial t}-4 x_{i} \frac{\partial^{2}}{\partial y_{i} \partial t}+4\left(x_{i}{ }^{2}+y_{i}{ }^{2}\right) \frac{\partial^{2}}{\partial t^{2}}\right)
$$

The family $\left\{X_{1}, \cdots, X_{n}, Y_{1}, \cdots Y_{n}\right\}$ satisfies Hörmander's rank condition ([19]) which implies that $\Delta_{\mathbb{H}}$ is hypoelliptic and the maximum principle is hold for the solutions to the equation including $\Delta_{\mathbb{H}}$ ([20]).

The integer $Q=2 n+2$ is called the homogeneous dimension of $\mathbb{H}^{n}$. Denote by $|\xi|_{\mathbb{H}}$ the distance from $\xi$ to the origin $(0,0,0)$ in $\mathbb{H}^{n}$ (see [8])

$$
|\xi|_{\mathbb{H}}=\left(\sum_{i=1}^{n}\left(x_{i}^{2}+y_{i}^{2}\right)^{2}+t^{2}\right)^{\frac{1}{4}}
$$

Authors in [14] used the norm

$$
|(z, w)|=\left(\sum_{i=1}^{n}\left(x_{i}^{2}+y_{i}^{2}\right)^{2}+16 t^{2}\right)^{\frac{1}{4}} \text { for }(x, y, t):=(z, w) \in \mathbb{H}^{n},
$$

which is equivalent to (2.5). The distance between two points $\xi$ and $\eta$ of $\mathbb{H}^{n}$ is defined by

$$
d_{\mathbb{H}}(\xi, \eta):=\left|\eta^{-1} \circ \xi\right|_{\mathbb{H}^{\prime}}
$$

where $\eta^{-1}$ denotes the inverse of $\eta$ with respect to $\circ$, that is $\eta^{-1}=-\eta$. The open ball of radius $R>0$ centered at $\xi$ is the set

$$
B_{\mathbb{H}}(\xi, R)=\left\{\eta \in \mathbb{H}^{n} \mid d_{\mathbb{H}}(\eta, \xi)<R\right\}
$$

It finds that $\xi \rightarrow|\xi|_{\mathbb{H}}$ is homogeneous of degree one with respect to $\delta_{\kappa}$ and

$$
\left|B_{\mathbb{H}}(\xi, R)\right|=\left|B_{\mathbb{H}}(0, R)\right|=\left|B_{\mathbb{H}}(0,1)\right| R^{Q},
$$

where $|\cdot|$ denotes the Lebesgue measure.

A function $u$ is called the cylindrical function, if

$$
u(x, y, t)=u(r, t)
$$

where $(x, y, t) \in \mathbb{H}^{n}$ and $r=\left(|x|^{2}+|y|^{2}\right)^{\frac{1}{2}}$.

Now we list three maximum principles that have been proved in [5], which will be used in the moving planes. 
Lemma 2.1. Let $\Omega$ be a bounded domain in $\mathbb{H}^{n}$ and suppose that $u \in L_{2 s}(\Omega) \cap C_{\text {loc }}^{1,1}(\Omega)$ is lower semicontinuous on $\bar{\Omega}$ satisfying

$$
\begin{cases}\left(-\Delta_{\mathbb{H}}\right)^{s} u(\xi) \geq 0 & \xi \in \Omega, \\ u(\xi) \geq 0 & \xi \in \mathbb{H}^{n} \backslash \Omega,\end{cases}
$$

then

$$
u(\xi) \geq 0, \quad \xi \in \Omega .
$$

Furthermore, if $u=0$ at some point in $\Omega$, then

$$
u(\xi)=0 \text {, almost everywhere in } \mathbb{H}^{n} .
$$

These conclusions also hold on the unbounded region $\Omega$, if we further assume $\varliminf_{|\xi|_{H_{H} \rightarrow \infty}} u(\xi) \geq 0$.

Denote by $\tilde{\xi}=(y, x, 2 \lambda-t)$ the $H$-reflection (see [23]) of $\xi=(x, y, t)$ about the plane $T_{\lambda}=\left\{\xi \in \mathbb{H}^{n} \mid t=\lambda, \lambda \in \mathbb{R}\right\}$ and let $H=\left\{\xi \in \mathbb{H}^{n} \mid t<\lambda\right\}$.

Lemma 2.2. Let $\Omega$ be a bounded domain in $H$. If the cylindrical function $u \in L_{2 s}(\Omega) \cap C_{\text {loc }}^{1,1}(\Omega)$ is lower semicontinuous on $\bar{\Omega}$ satisfying

$$
\begin{cases}\left(-\Delta_{\mathbb{H}}\right)^{s} u(\xi) \geq 0 & \xi \in \Omega, \\ u(\xi) \geq 0 & \xi \in H \backslash \Omega, \\ u(\tilde{\xi})=-u(\xi) & \xi \in H,\end{cases}
$$

then

$$
u(\xi) \geq 0, \quad \xi \in \Omega .
$$

Furthermore, if $u=0$ at some point in $\Omega$, then

$$
u(\xi)=0 \text {, almost everywhere in } \mathbb{H}^{n} .
$$

These conclusions also hold on the unbounded region $\Omega$, if we further assume $\varliminf_{|\xi|_{\mathrm{H}} \rightarrow \infty} u(\xi) \geq 0$.

Lemma 2.3. Let $\Omega$ be a bounded narrow domain in $H$ and locate in the domain $\{\xi \mid \lambda-l<t<\lambda\}$ for small $l$. Assume that $c(x)$ is bounded from below in $\Omega$ the cylindrical function $u \in L_{2 s}(\Omega) \cap$ $C_{\text {loc }}^{1,1}(\Omega)$ is lower semicontinuous on $\bar{\Omega}$ and satisfies

$$
\begin{cases}\left(-\Delta_{\mathbb{H}}\right)^{s} u(\xi)+c(\xi) u(\xi) \geq 0 & \xi \in \Omega, \\ u(\xi) \geq 0 & \xi \in H \backslash \Omega, \\ u(\tilde{\xi})=-u(\xi) & \xi \in H,\end{cases}
$$

then

$$
u(\xi) \geq 0, \quad \xi \in \Omega
$$


Furthermore, if $u=0$ at some point in $\Omega$, then

$$
u(\xi)=0 \text {, almost everywhere in } \mathbb{H}^{n} .
$$

These conclusions also hold on an unbounded region $\Omega$, if we further assume $\varliminf_{|\xi|_{\mathrm{H}} \rightarrow \infty} u(\xi) \geq 0$.

\section{Proof of Theorem 1.1}

We will use Lemmas 2.1, 2.2 and 2.3 to prove Theorem 1.1.

Proof. By the condition (1.7), we claim that

$$
u(\xi)>0 \text { or } u(\xi) \equiv 0, \text { for any } \xi \in \mathbb{H}_{+}^{n} .
$$

In fact, suppose that $u(\xi)>0$ is not right, we will show $u(\xi) \equiv 0$. The lower semicontinuousity of $u$ on $\mathbb{H}_{+}^{n}$ implies that there exists $\xi^{0} \in \overline{\mathbb{H}}_{+}^{n}$, such that

$$
u\left(\xi^{0}\right)=\min _{\mathbb{H}_{+}^{n}} u=0,
$$

then

$$
\begin{aligned}
\left(-\Delta_{\mathbb{H}}\right)^{s} u\left(\xi^{0}\right) & =C_{Q, s} P V \int_{\mathbb{H}^{n}} \frac{u\left(\xi^{0}\right)-u(\eta)}{\left|\eta^{-1} \circ \xi^{0}\right|_{\mathbb{H}} Q+2 s} \mathrm{~d} \eta \\
& =C_{Q, s} P V \int_{\mathbb{H}_{+}^{n}} \frac{u\left(\xi^{0}\right)-u(\eta)}{\left|\eta^{-1} \circ \xi^{0}\right|_{\mathbb{H}} Q+2 s} \mathrm{~d} \eta \\
& =C_{Q, s} P V \int_{\mathbb{H}_{+}^{n}} \frac{-u(\eta)}{\left|\eta^{-1} \circ \xi^{0}\right|_{\mathbb{H}} Q+2 s} \mathrm{~d} \eta \\
& =u^{p}\left(\xi^{0}\right)=0 .
\end{aligned}
$$

Hence

$$
\int_{\mathbb{H}_{+}^{n}} \frac{-u(\eta)}{\left|\eta^{-1} \circ \xi^{0}\right|_{\mathbb{H}}^{Q+2 s}} \mathrm{~d} \eta=0,
$$

and so $u(\xi) \equiv 0$ for any $\xi \in \mathbb{H}_{+}^{n}$.

In the sequel, we only need to treat the case $u>0$ on $\mathbb{H}_{+}^{n}$. Let us use the method of moving planes to $u$ along the $t$ direction and denote

$$
\begin{aligned}
& T_{\lambda}^{+}=\left\{\xi \in \mathbb{H}_{+}^{n} \mid t=\lambda, \lambda \in \mathbb{R}^{+}\right\}, \\
& \Sigma_{\lambda}^{+}=\left\{\xi \in \mathbb{H}_{+}^{n} \mid 0<t<\lambda\right\} .
\end{aligned}
$$

Letting

$$
u_{\lambda}(\xi)=u_{\lambda}(|(x, y)|, t):=u(|(x, y)|, 2 \lambda-t)
$$


and using the $H$-refection, we have

$$
u_{\lambda}(\xi)=u(y, x, 2 \lambda-t)=u\left(\xi^{\lambda}\right)
$$

Denote

$$
w_{\lambda}(\xi)=u_{\lambda}(\xi)-u(\xi)
$$

If $\lambda>0$ is sufficiently small, we use Lemma 2.3 to the narrow region $\Omega=\Sigma_{\lambda}^{+}$in $\Sigma_{\lambda}=$ $\Sigma_{\lambda}^{+} \cup\left(\mathbb{H}^{n} \backslash \mathbb{H}_{+}^{n}\right)$, to have

$$
w_{\lambda}(\xi) \geq 0,
$$

which provides the start point of moving planes. Now we will explain that the plane $T_{\lambda}^{+}$ can be moved to the infinite such that (3.1) holds. Concretely, letting

$$
\lambda_{0}=\sup \left\{\lambda>0 \mid w_{\mu}(\xi) \geq 0, \forall \xi \in \Sigma_{\lambda}^{+}, \mu \leq \lambda\right\}
$$

we will prove

$$
\lambda_{0}=\infty
$$

To prove (3.2), let us consider $\lambda_{0}<\infty$, which implies contradiction. In fact, if $\lambda_{0}$ sufficiently big, we only to show that

$$
w_{\lambda}(\xi) \geq 0, \quad \xi \in \Sigma_{\lambda}^{+}
$$

Note that (1.7) implies that there exist a point $\xi \in \Sigma_{\lambda}^{+}$such that

$$
w_{\lambda}\left(\xi^{0}\right)=\min _{\Sigma_{\lambda}^{+}} w_{\lambda}<0,
$$

i.e. $u_{\lambda}\left(\xi^{0}\right) \leq \zeta_{\lambda}\left(\xi^{0}\right) \leq u\left(\xi^{0}\right)$. By (1.6), we have

$$
\left(-\Delta_{\mathbb{H}}\right)^{s} w_{\lambda}(\xi)=p\left(\zeta_{\lambda}(\xi)\right)^{p} w_{\lambda}(\xi),
$$

where $\zeta_{\lambda}(\xi)$ is between $u_{\lambda}(\xi)$ and $u(\xi)$. For $\lambda \rightarrow \infty$, by (1.7), $u(\xi) \rightarrow 0$ and $u_{\lambda}(\xi) \rightarrow 0$, hence so is to $\zeta_{\lambda}(\xi)$. Then

$$
\left(-\Delta_{\mathbb{H}}\right)^{s} w_{\lambda}\left(\widetilde{\zeta}^{0}\right)<0,
$$

which contradict the fact that $\xi^{0}$ is a negative minimum of $w_{\lambda}$, hence (3.3) and so (3.2) are proved, and also $\left|\xi^{0}\right|_{\mathbb{H}}$ must be bounded.

If $\lambda_{0}<\infty$, then we can derive that $T_{\lambda}^{+}$can be moved further to the right, that is, we have claim

there exists $\sigma>0$, such that $w_{\lambda}(\xi) \geq 0$ for any $\lambda \in\left(\lambda_{0}, \lambda_{0}+\sigma\right)$. 
This will contradict to the definition of $\lambda_{0}$ and so (3.2) holds.

In the sequel, we prove the claim. If $|\xi|_{\mathbb{H}}\left(\xi\right.$ is the negative minimum of $\left.w_{\lambda}(\xi)\right)$ sufficiently large, then the above result that $|\xi|_{\mathbb{H}}$ must be bounded implies that there exists some $R_{0}>0$, such that $w_{\lambda}(\xi) \geq 0$ on $\mathbb{H}_{+}^{n} \backslash B_{\mathbb{H}}\left(0, R_{0}\right)$. Next we point out that $w_{\lambda}(\xi) \geq 0$ is also true on $B_{\mathbb{H}}\left(0, R_{0}\right)$. To see it, we note $\lambda_{0}<\infty$ and get by Lemma 2.2 that on $\xi \in \Sigma_{\lambda_{0}}^{+} \cap B_{\mathbb{H}}\left(0, R_{0}\right)$,

$$
w_{\lambda_{0}}(\xi)>0
$$

or

$$
w_{\lambda_{0}}(\xi) \equiv 0
$$

In the case $w_{\lambda_{0}}(\xi) \equiv 0$, we observe $u(\xi) \equiv 0$ by the boundary conditions of $u$. On the other hand, (3.4) implies that there exists a small $\delta>0$, such that

$$
w_{\lambda_{0}}(\xi) \geq c>0, \quad \xi \in \overline{\Sigma_{\lambda_{0}-\delta}^{+} \cap B_{\mathbb{H}}\left(0, R_{0}\right)} .
$$

Since $w_{\lambda}$ relies continuously on $\lambda$, there exists $\varepsilon, 0<\varepsilon<\sigma$, such that

$$
w_{\lambda_{0}+\varepsilon}(\xi) \geq 0, \quad \xi \in \overline{\Sigma_{\lambda_{0}-\delta}^{+} \cap B_{\mathbb{H}}\left(0, R_{0}\right)} .
$$

Noting $\left(\Sigma_{\lambda_{0}+\varepsilon}^{+} \mid \Sigma_{\lambda_{0}-\delta}^{+}\right) \cap B_{\mathbb{H}}\left(0, R_{0}\right)$ is a narrow region, we have by Lemma 2.3,

$$
w_{\lambda_{0}+\varepsilon}(\xi) \geq 0, \quad \xi \in \overline{\Sigma_{\lambda_{0}+\varepsilon}^{+} \cap B_{\mathbb{H}}\left(0, R_{0}\right)} .
$$

Therefore the claim is shown.

Using (3.2) and Lemma 2.2 once again, it yields for any $\xi \in \Sigma_{\lambda}^{+}$,

$$
w_{\lambda}(\xi)>0
$$

or

$$
w_{\lambda}(\xi) \equiv 0
$$

To (3.8), we have $u(\xi) \equiv 0$. From (3.7), we know that $u(\xi)$ is strictly increasing, which contradicts to the boundary condition of $u$ and (1.7). Hence, we also have $u(\xi) \equiv 0$.

\section{Proof of Theorem 1.2}

The proof of Theorem 1.2 follows the idea in $[4,6]$, and the process of proof of Theorem 1.1. We state some notations.

Let $T_{\lambda}$ be a hyperplane in $\mathbb{H}^{n}$ defined by

$$
T_{\lambda}=\left\{\xi \in \mathbb{H}^{n} \mid t=\lambda, \lambda \in \mathbb{R}\right\} .
$$

Denote the H-reflection of $\xi=(x, y, t)$ about the plane $T_{\lambda}$ by $\tilde{\xi}=(y, x, 2 \lambda-t)$, and the region in the left of the plane $T_{\lambda}$ by

$$
\Sigma_{\lambda}=\left\{\xi \in \mathbb{H}^{n} \mid t<\lambda\right\} .
$$

The definition of $u_{\lambda}$ and $w_{\lambda}$ can refer to Section 3 . 
Proof. First we will check that for $\lambda$ sufficiently negative, it holds

$$
w_{\lambda}(\xi) \geq 0, \quad \xi \in \Sigma_{\lambda}
$$

Indeed, suppose that (4.1) is violated, then by (1.7), there exists a point $\xi^{0} \in \Sigma_{\lambda}$ such that

$$
w_{\lambda}\left(\xi^{0}\right)=\min _{\Sigma_{\lambda}} w_{\lambda}<0,
$$

i.e. $u_{\lambda}\left(\xi^{0}\right) \leq \varsigma_{\lambda}\left(\xi^{0}\right) \leq u\left(\xi^{0}\right)$. Note by $(1.2)$ that

$$
\left(-\Delta_{\mathbb{H}}\right)^{s} u_{\lambda}(\xi)-\left(-\Delta_{\mathbb{H}}\right)^{s} u(\xi)=f^{\prime}\left(\varsigma_{\lambda}(\xi)\right) w_{\lambda}(\xi)=C w_{\lambda}(\xi),
$$

where $\varsigma_{\lambda}(\xi)$ is between $u_{\lambda}(\xi)$ and $u(\xi)$. For sufficiently negative $\lambda, u\left(\xi^{0}\right) \rightarrow 0$ by (1.7), so is $\varsigma_{\lambda}\left(\xi^{0}\right)$. From (4.2), and Lemma 2.2, a contradiction is obtained and hence (4.1) is proved.

The above result provides the starting point of moving planes. Let us move the plane $T_{\lambda}$ to the right as long as (4.1) holds to its limiting position

$$
\lambda_{0}=\sup \left\{\lambda \mid w_{\mu}(\xi) \geq 0, \quad \forall \xi \in \Sigma_{\mu}, \mu \leq \lambda\right\} .
$$

We will show that

$$
w_{\lambda_{0}}(\xi)=0, \quad \xi \in \Sigma_{\lambda_{0}} .
$$

In fact, by Lemmas 2.2 and 2.3, as in the proof of Theorem 1.1, we can show that

$$
w_{\lambda_{0}}(\xi) \geq 0, \quad \xi \in \Sigma_{\lambda_{0}}
$$

is holds.

Similarly, we can move the plane from $+\infty$ to the left to get

$$
w_{\lambda_{0}}(\xi) \leq 0, \quad \xi \in \Sigma_{\lambda_{0}} .
$$

Combining (4.4) and (4.5), it derives (4.3).

Hence, we see that $u$ must be symmetric and monotone with respect to $t$ about some point.

\section{Acknowledgement}

This work is supported by the National Natural Science Foundation of China (Grant No. 11771354) and the National Natural Science Basic Research plan in Shaanxi Province of China (Grant No. 2016JM1023). 


\section{References}

[1] Caffarelli L. and Silvestre L., An extension problem related to the fractional Laplacian. Comm. Partial Differential Equations, 2 (2007), 1245-1260.

[2] Brändle C., Colorado E., de Pablo A. and Sánchez U., A concaveconvex elliptic problem involving the fractional Laplacian. Proc Royal Soc. of Edinburgh, 143 (2013), 39-71.

[3] Chen W. and Li C., Maximum principles for the fractional p-Laplacian and symmetry of solutions. 2017, arXiv:1705.04891v1.

[4] Chen W., Li C. and Li Y., A direct method of moving planes for the fractional Laplacian. Adv. Math., 308 (2016), 404-437.

[5] Wang X. and Niu P., A direct method of moving planes for fractional power subLaplacian on the Heisenberg group, submited.

[6] Gidas B., Ni W.M. and Nirenberg L., Symmetry and related properties via the maximum principle. Comm. Math. Phys., 68 (1979), 209-243.

[7] Dou J., Niu P. and Yuan Z., A Hardy inequality with remainder terms in the Heisenberg group and the weighted eigenvalue problem. J. Inequal. Appl., 2007 (1) (2007), 1-24.

[8] Folland G.B. and Stein E.M., Estimates for the $\bar{\partial}_{b}$ complex and analysis on the Heisenberg group. Comm. Pure Appl. Math., 27 (4) (1974), 429-522.

[9] Garofalo N. and Lanconelli E., Existence and nonexistence results for semilinear equations on the Heisenberg group. Indiana Univ. Math. J., 41 (1992), 71-98.

[10] Jerison D., Boundary regularity in the dirichlet problem for $\square$ b on CR manifolds. Comm. Pure Appl. Math., 36 (2) (1983), 143-181.

[11] Jerison D.S. and Lee J.M., The Yamabe problem on CR manifolds. J. Diff. Geom., 25 (1987), 167-197.

[12] Ferrari F., Franchi B., Harnack inequality for fractional laplacians in Carnot groups. Math. Z., 279 (2015), 435-458.

[13] Frank R., Gonzalez M., Monticelli D. and Tan J., An extension problem for the CR fractional Laplacian. Adv. Math., 270 (2015), 97-137.

[14] Roncal L. and Thangavelu S., Hardy's inequality for fractional powers of the sublaplacian on the Heisenberg group. Adv. Math., 302 (2016), 106-158.

[15] Cowling M. and Haagerup U., Completely bounded multipliers of the Fourier algebra of a simple Lie group of real rank one. Invent. Math., 96 (1989), 507-549.

[16] Cinti E. and Tan J., A nonlinear Liouville theorem for fractional equations in the Heisenberg group. J. Math. Anal. Appl., 433 (2016), 434-454.

[17] Wang X., Niu P. and Cui X., A Liouville type theorem to an extension problem relating to the Heisenberg group. Commun. Pure Appl. Anal., 17 (6) (2018), 2379-2394.

[18] Chen W. and Li C., Methods on Nonlinear Elliptic Equations, AIMS book series, vol.4, 2010.

[19] Hörmander L., Hypoelliptic second order differential equations. Acta Math., 119 (1967), 147171.

[20] Bony J.M., Principe du Maximum, Inegalite de Harnack et unicite du probleme de Cauchy pour les operateurs ellipitiques degeneres. Ann. Inst. Fourier Grenobles, 19 (1969), 277-304.

[21] Birindelli I., Capuzzo Dolcetta I. and Cutrì A., Liouville theorems for semilinear equations on the Heisenberg group. Ann. Inst. H. Poincaré Anal. Non Linéaire, 14 (3) (1997), 295-308.

[22] Birindelli I. and Cutrì A., A semi-linear problem for the Heisenberg Laplacian. Rend. Sem. Mat. Della Univ. Padova, 94 (1995), 137-153.

[23] Birindelli I. and Prajapat J., Nonlinear Liouville theorems in the Heisenberg group via the 
moving plane method. Comm. Partial Diff. Eqs., 24 (9-10) (1999), 1875-1890.

[24] Birindelli I. and Prajapat J., Monotonicity and symmetry results for degenerate elliptic equations on nilpotent Lie groups. Pacific J. Math., 204 (1) (2002), 1-17.

[25] Bonfiglioli A., Lanconelli E. and Uguzzoni F., Stratified Lie groups and potential theory for their sub-Laplacians. Springer Monogr. Math., Springer, New York 2007.

[26] Chen W. and Li C., Classification of solutions of some nonlinear elliptic equations. Duke Math. J., 63 (3) (1991), 615-622.

[27] Chen W., Li C. and Ou B., Classification of solutions for an integral equation. Comm. Pure Appl. Math., 59 (3) (2006), 330-343.

[28] Folland G.B., Fundamental solution for subelliptic operators. Bull. Amer. Math. Soc., 79 (1979), 373-376.

[29] Gidas B. and Spruck J., Global and local behavior of positive solutions of nonlinear elliptic equations. Comm. Pure Appl. Math., 35 (1982), 528-598.

[30] Gilbarg D. and Trudinger N.S., Elliptic Partial Differential Equations of Second Order, Berlin: Springer-Verlage, 1983.

[31] Helms L.L., Introduction to Potential Theory, Pure and Applied Mathematics 22, WileyInterscience, New York, London, Sydney, 1969.

[32] Landkof N.S., Foundations of Modern Potential Theory, Springer-Verlag Berlin Heidelberg, New York, 1972. Translated from the Russian by A. P. Doohovskoy, Die Grundlehren der mathematischen Wissenschaften, Band 180.

[33] Li Y. and Ni W.M., Radial symmetry of positive solutions of nonlinear elliptic equations in $R^{n}$. Comm. Partial Diff. Eqs., 18 (1993), 1043-1054. 